Revista de Economia Política, vol. 30, no 2 (118), pp. 340-356, abril-junho/2010

\title{
A teoria da perspectiva e as mudanças de preferência no mainstream: um prospecto Lakatoseano
}

\author{
BRUNO BERGER* \\ HUÁSCAR FIALHO PESSALI**
}

The theory of the perspective and the changes of preference in the mainstream: a Lakatosean prospect. For many decades over the 20th Century, the mainstream of economics adopted a normative and axiomatic theory of individual behavior in which maximizing procedures were carried out by rationally unbounded agents. This status has been challenged on many grounds and alternative views from fields like psychology have found a way into the core of economics research frontier. Prospect theory, developed by psychologists Daniel Kahneman and Amos Tversky since the 1970s, has provided a more empirical, inductive and descriptive theory of decision making. It has made significant inroads into mainstream microeconomics, shaking the habits of some of its practitioners. This paper first takes stock of its main developments and then uses a Lakatosian framework to draw out its negative and positive heuristics. In what follows, its heuristics are compared to those of traditional rational decision-making theories. The differences between them are highlighted, pointing to changes in the mainstream of the profession and to new opportunities for research.

Keywords: decision making; prospect theory; behavioral economics; experimental economics; expected utility theory.

JEL Classification: D01; B41.

\section{INTRODUÇÃO}

O mainstream econômico não é mais o mesmo. Outros programas de pesquisa têm ocupado o espaço antes ocupado pela teoria neoclássica (Colander, 2000; Colan-

\footnotetext{
* Programa de Pós-Graduação em Desenvolvimento Econômico da UFPR, e-mail: brunoberger85@gmail. com.

** Departamento de Economia e Programa de Pós-Graduação em Desenvolvimento Econômico da UFPR, pessali@ufpr.br. Submetido: Fevereiro 2008; Aprovado: Janeiro 2009.
} 
der et al. 2004; Davis, 2006, 2008a,b; Dequech, 2007; Friedman, 1998; Hodgson 2007a). Isso acontece, por exemplo, em um tema caro ao neoclassicismo: a teorização sobre o processo decisório dos indivíduos. Afinal, as decisões individuais de consumidores, poupadores e investidores resultam em última instância no comportamento de quase todas as variáveis econômicas, sejam micro, meso ou macroeconômicas.

As suposições neoclássicas a respeito do processo decisório - indivíduos dotados de racionalidade substantiva cujo comportamento é consistente na maximização de sua utilidade, dada uma função de preferências estáveis - compõem o núcleo duro da teoria e tornaram-se parte do mainstream econômico ao longo do século XX. Thorstein Veblen (1898) as havia criticado ainda no século XIX, mas só a partir da década de 1950 algumas proposições oriundas de outras ciências conseguiram maior contundência. Seu impacto se avolumou e conseguiu mudar a feição do mainstream a partir da década de 1980 (Colander et al. 2004; Davis, 2006), principalmente com ideias vindas de outra ciência com ênfase no indivíduo per se - a psicologia. A partir da década de 1950 uma área da psicologia se tornou especialmente propícia ao interesse da economia, a Behavioral Decision Research — BDR (ver Payne \& Bettman, 1992). Que economistas reconheçam nomes como os de Herbert Simon, Ward Edwards e Leonard Savage é em grande parte resultado de seus desdobramentos (ver Sent, 2004). Que reconheçam nomes como os dos psicólogos Daniel Kahneman e Amos Tversky e rendam ao primeiro o Prêmio Nobel de Economia em 2002 é em grande parte resultado da fertilidade e longevidade de seus insights. A extensão de tal reconhecimento pode ser ilustrada pelas citações feitas a Kahneman tanto na psicologia quanto na economia (Figura 1). É aparente ali um efeito positivo do Prêmio Nobel sobre o volume de citações anuais recebidas na forma de uma mudança persistente de inclinação na série. ${ }^{1}$

Figura 1: Citações ao trabalho de Daniel Kahneman 1966-2007

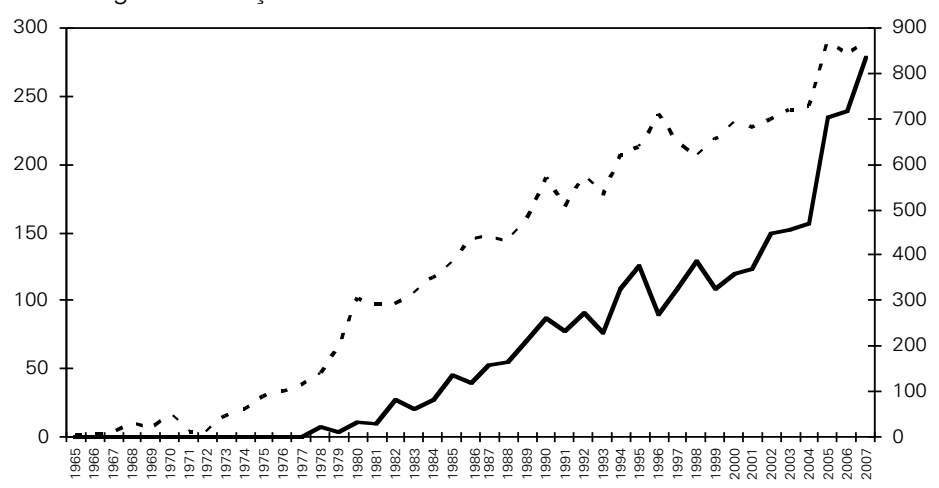

Economia

- - - Psicologia

Fonte: Social Science Citation Index, versão eletrônica, vários anos.

\footnotetext{
${ }^{1}$ A taxa média de acréscimos de citações nos cinco anos posteriores a 2002, por exemplo, é duas vezes maior que a mesma taxa para os cinco anos anteriores a 2002 (inclusive).
} 
Embora o conjunto de influências da psicologia sobre a economia a partir da BDR seja heterogêneo, em geral o reconhecemos pelo nome de economia comportamental. Dentro deste conjunto encontramos a teoria da perspectiva, formulada a partir da década de 1970 por Kahneman e Tversky. O espaço por eles obtido e seu explícito desafio aos chamados modelos normativos de escolha do mainstream econômico são os principais motivadores de sua escolha como objeto de análise neste trabalho. A tarefa a que nos lançamos aqui é sugerir uma identificação metodológica da teoria da perspectiva a partir do esquema núcleo duro-cinturão de proteção proposto por Imro Lakatos. Em fazendo isto, tentamos discutir alguns desdobramentos possíveis para a prática acadêmica da economia.

Para fins de contextualização, começamos por fazer uma breve apresentação da teoria da perspectiva e, em seguida, da prática experimental que a caracteriza. No que segue, fazemos uma caracterização dos modelos racionais de tomada de decisão e da teoria da perspectiva usando um esquema analítico lakatoseano. O uso deste esquema analítico tem o intuito mais de identificar os sistemas teóricos em questão do que de determinar uma trajetória de evolução da ciência de um programa de pesquisa para o outro, ou outros. Nosso propósito é identificar melhor a participação da teoria da perspectiva num momento de aparente mudança na ciência econômica, e cujo resultado final não se faz previsível. Algumas considerações finais fecham o ensaio.

\section{O DESENVOLVIMENTO DA TEORIA DA PERSPECTIVA}

As pesquisas e discussões que nutriram a teoria da perspectiva tiveram início na década de 1950. Naquela década Maurice Allais delineou uma teoria da escolha sob incerteza baseada na psicologia, Ward Edwards introduziu a tomada de decisão como um tópico de pesquisa para os psicólogos, e Herbert Simon deu grande fôlego à noção de racionalidade limitada. Um dos desdobramentos de tais iniciativas foi a pesquisa em psicologia cognitiva. A psicologia cognitiva tomou impulso com os resultados de pesquisa de Daniel Kahneman e Amos Tversky publicados em periódicos de psicologia a partir de 1971.

Em 1974 eles publicaram um artigo na revista Science com o título "Judgment under uncertainty" (Tversky \& Kahneman, 1974). Utilizando o resultado de diversos experimentos, eles afirmam que as pessoas utilizam um número limitado de heurísticas para reduzir a complexidade de determinar probabilidades e prever resultados ao tomarem suas decisões. Este estudo foi um trabalho de natureza mais ampla sobre os processos cognitivos e não voltado especificamente para a economia. Mas a discussão tem grande afinidade com o tema "escolha sob risco", que é de grande apreço aos economistas, e seus resultados sugeriam que o julgamento sob incerteza se desviava sistematicamente da racionalidade substantiva adotada pelo mainstream econômico de então. Tais padrões detectados serviram de base indutiva para a formulação das hipóteses teóricas do que viria a ser conhecida como teoria da perspectiva (prospect theory). 


\section{A teoria da perspectiva na economia}

Kahneman e Tversky retribuiram o interesse dos economistas publicando um artigo de grande repercussão na Econometrica (Kahneman \& Tversky, 1979). Neste artigo, que se tornou o mais citado de todos os já publicados na Econometrica (RSAS, 2002), os autores apresentaram uma crítica aberta à (in)capacidade descritiva dos modelos de utilidade esperada. ${ }^{2}$ Eles argumentaram que em escolhas com prospectos de risco, os agentes demonstravam padrões de comportamento inconsistentes com os preceitos básicos daquela teoria, e propuseram a teoria da perspectiva como uma teoria alternativa da escolha.

A este artigo sucederam outros trabalhos em periódicos de economia. Kahneman e Tversky (1986), por exemplo, argumenta que as teorias racionais de escolha sob risco e incerteza, como a teoria dos jogos ou a teoria de alocação de portfólio, foram pensadas como modelos nos quais um agente idealizado toma suas decisões. Ou seja, o comportamento do agente é definido a priori nas especificações do modelo. Por isso tais teorias são normativas: elaboram um sistema lógico (às vezes totalmente axiomático) para estabelecer como um agente deve tomar decisões levando em conta possíveis resultados e seus riscos, de modo que os modelos tenham resultados inequívocos. Apesar de seu caráter normativo, essas teorias e modelos são usados na tentativa de predizer o comportamento das pessoas e, por vezes, para recomendar cursos de ação real (Kahneman \& Tversky, 1986). A partir disto, os autores viram um trade-off entre as proposições neoclássicas e as comportamentais: um modelo ou teoria de escolha não pode ser ao mesmo tempo normativamente adequado e descritivamente válido.

Para Kahneman e Tversky (1986), os modelos normativos têm seu mérito como tal, mas não fornecem uma base válida para explicar o comportamento dos agentes. Para tanto, é preciso uma teoria descritiva que busque inferir como as pessoas tomam decisões, independente de serem ou não ótimas. Ao se fazer isso, discrepâncias certamente serão identificadas no comportamento dos agentes, ou seja, inconsistências com as teorias normativas. De acordo com os autores, estas anomalias são difundidas demais para serem ignoradas, sistemáticas demais para serem tratadas como erros aleatórios, e fundamentais demais para serem acomodadas relaxando o sistema normativo.

Tversky e Kahneman (1992) extende a teoria original, chamando-a de teoria da perspectiva cumulativa. Há ali uma formalização mais robusta e um esforço maior de ampliar sua capacidade preditiva, tentando satisfazer duas demandas importantes de boa parte da comunidade de economistas.

Além dos próprios trabalhos de Kahneman e Tversky, outras frentes de pesquisa se abriram com a teoria da perspectiva. ${ }^{3}$ Camerer (1998, pp. 17-18) mencio-

\footnotetext{
${ }^{2}$ Uma consulta feita em janeiro de 2009 ao Social Science Citation Index <http://isiwebofknowledge. com> contabilizou 5.631 citações ao artigo.

${ }^{3}$ Ver Kahneman \& Tversky (2000) para uma amostra de seus desenvolvimentos.
} 
na algumas delas na área da tomada de decisão, como a conjunção com a ciência da computação. Isto tem permitido simular modelos em que limites à racionalidade dos agentes são aplicados, gerando resultados complexos e similares aos vistos na realidade. Outra área com que a teoria da perspectiva troca insights é a neuroeconomia, que utiliza técnicas de imagem neural e ferramentas psicofisiológicas para identificação empírica de preferências dos indivíduos (Glimcher, 2002; Hands 2007; Villeval, 2007). Não se pode ser exaustivo aqui, nem pretender sugerir que tais áreas são apenas frutos diretos da teoria da perspectiva. Mas sua visibilidade na crítica dos modelos racionais de tomada de decisão, influenciou e em alguns casos possibilitou a difusão de ideias externas à economia neste respeito.

Outro aspecto relevante da teoria da perspectiva é a sua base experimental. Vindos da psicologia, área em que experimentos são costumeiramente disseminados, Kahneman e Tversky não se preocupam em discutir a validade do método. ${ }^{4}$ Ainda assim, sua prática parece ter somado credibilidade ao método dentro da economia como fonte de evidências empíricas - ao menos na área de comportamento humano e tomada de decisão.

\section{A teoria da perspectiva e os experimentos em economia}

Vernon Smith (1982), que dividiu o Nobel de 2002 com Kahneman, caracteriza um experimento nas ciências econômicas como a reprodução de um sistema microeconômico definido por dois componentes: ambiente e instituições. O ambiente é definido por informações como o número de agentes participantes, suas preferências, os bens existentes, a posse de tecnologia ou conhecimento e a posse dos bens. Ou seja, o ambiente microeconômico é delimitado por uma gama de características estáveis do contexto de ação. Já as instituições são as "regras do jogo" - o que é ou não permitido nos experimentos, os meios de comunicação, as maneiras de realização das trocas, e outras especificações (Smith, 1982). Estas são as variáveis de controle do experimentador para que se observe um terceiro componente do experimento, o comportamento dos participantes, que resulta de sua interação com o ambiente e as instituições.

Para que se possa controlar os dois componentes acima e para que os resultados do experimento tenham validade fora do laboratório, cinco condições precisam ser atendidas: não saciamento (nonsatiation), saliência (saliency), dominância (dominance), privacidade (privacy) e paralelismo (parallelism). Para Smith (1982), as quatro primeiras condições garantem a existência de um experimento microeconômico controlado, permitindo o teste de hipóteses e teorias. A quinta condição, o paralelismo, refere-se à possibilidade de se transferir os resultados de um experi-

\footnotetext{
${ }^{4}$ Essa discussão não nos cabe aqui. Para o leitor interessado no tema, sugerimos Smith (1982, 2002), Kagel e Roth (1995), Siakantaris (2000), Bianchi (2001), Guala (2002) e Villeval (2007). Vale ressaltar que experimentos também são usados em outras áreas da economia sem interesses primordialmente comportamentais.
} 
mento em laboratório para fora dele. Isto permite a geração de hipóteses através de resultados de experimentos. ${ }^{5}$

Da condição de paralelismo surgem as principais críticas à utilização do método experimental na economia. Guala (2002) ressalta que o próprio progresso da economia experimental permite que isso seja discutido sob uma perspectiva empírica - o sucesso ou não de exportar os resultados de laboratório para situações reais de decisão na economia vai determinar se são mais ou menos válidos e em que áreas específicas podem ser utilizados. Em outras palavras, a discussão ex-ante sobre a validade do método é relevante mas não conclusiva ${ }^{6}$

Kahneman e Tversky em particular, utilizam experimentos cujo objeto mais pontual é a tomada de decisão individual. Assim, seus experimentos em geral não forçam as quatro primeiras condições. A condição de paralelismo, porém, merece mais atenção em função do caráter descritivo atribuído pelos autores à teoria da perspectiva, ou seja, seus experimentos visam testar e gerar hipóteses teóricas.

Os experimentos de Kahneman e Tversky objetivam, a princípio, falsear os pressupostos de caráter universal dos modelos racionais de escolha (alguns exemplos estão em Kahneman \& Tversky, 1986)..$^{7}$ A questão do paralelismo não parece problemática aqui: o experimento, mesmo feito em laboratório, continua sendo uma escolha real, feita por um agente real, com incentivos e riscos reais. A não corroboração de tais pressupostos por um dos indivíduos participantes traz, assim, o falseamento de sua universalidade (Siakantaris, 2000).

Quanto a gerar hipóteses sobre o comportamento e capacidade cognitiva dos agentes, a própria possibilidade de realizar experimentos permite verificar a validade de seus resultados (Guala, 2002). Sua replicação ou ainda a realização de outros testes que confirmem ou neguem os resultados obtidos é o que permitirá ponderar a validade dos mesmos.

\section{O MAINSTREAM EM PERSPECTIVA: UMA ANÁLISE METODOLÓGICA}

O espaço ganho pela teoria da perspectiva nos círculos acadêmicos da economia evidencia uma mudança. Talvez isso venha a ser reconhecido como uma revolução científica à la Kuhn. Neste momento, porém, é difícil parametrizar a velocidade da mudança, afirmar sua irreversibilidade ou ter certeza de que se dará de forma linear em prol de um paradigma experimental da construção de teorias econômicas em que a teoria da perspectiva teria uma posição central. ${ }^{8} \mathrm{O}$ que se pode perceber são

\footnotetext{
${ }^{5}$ Uma análise mais extensa destas condições é feita por Smith (1982).

${ }^{6}$ Essa discussão está além do escopo deste trabalho. Para aprofundamentos no tema, ver Smith (1982, 2002), Siakantaris (2000), Bianchi (2001), Guala (2002) e Villeval (2007).

${ }^{7}$ Muito embora seja sempre possível usá-los na explicação post hoc de quaisquer resultados, tornando-a não falseável (Hutchison, 1938; Boland, 1981; Hodgson, 2004).

${ }^{8}$ Plott (1991) lida com essa questão mais ampla.
} 
sinais de mudança em andamento. Já reportamos o Nobel de Kahneman e Smith como um deles, bem como as crescentes citações em economia ao trabalho de Kahneman. O espaço ocupado pelo economista Richard Thaler, colaborador de Kahneman e Tversky, no Journal of Economic Perspectives com sua seção de "anomalias" comportamentais também pode ser visto como uma evidência. ${ }^{9} \mathrm{E}$ a todos estes sinais podemos acrescentar o diagnóstico de vários autores em posição privilegiada na profissão. ${ }^{10}$ Diante disto, o que se tenta aqui é, à la Lakatos, delinear os elementos teóricos em jogo nos moldes de programas de pesquisa com conformações distintas. A partir daí, tenta-se explorar um pouco a relação entre a conformação neoclássica e a da teoria da perspectiva, com destaque para suas diferenças. Isto nos permitirá especular um pouco sobre algumas mudanças recentes nas preferências, i.e., na prática observada do mainstream econômico.

Comecemos por relembrar um pouco de Lakatos. Para ele, as ciências se desenvolvem transferindo de forma progressiva e degenerativa os problemas que levanta e trata, e esta dinâmica se dá a partir de programas de pesquisa. Tais programas possuem regras metodológicas a ditar que caminhos seguir ou evitar (Lakatos, 1979). Estas regras metodológicas se dividem em heurísticas negativa e positiva. A heurística negativa, ou o "núcleo" do programa, é a parte do programa tida como irrefutável por uma decisão metodológica a priori. Deste conjunto de leis ou ideias são extraídos hipóteses auxiliares e modelos que simulam em algum grau a realidade. Estes perfazem a heurística positiva - um conjunto de hipóteses auxiliares que serve de "cinturão de proteção" para o núcleo do programa ao ser exposto a testes empíricos (Lakatos, 1979).

A refutação de uma hipótese empírica ou metafísica que faz parte de um cinturão protetor, porém, por si só não significa a derrocada de determinado programa de pesquisa. Observar uma falha do programa para explicar determinado fenômeno (o que pode ser chamado de "anomalia") não quer dizer que o núcleo é falso ou incompleto. Por vezes a observação de anomalias pode se tornar justamente o trunfo de um projeto de pesquisa se sua heurística negativa conseguir gerar novas hipóteses ou modelos auxiliares que expliquem tanto o que já era explicado quanto as anomalias constatadas (Lakatos, 1979). Entretanto, se o núcleo duro não consegue adaptar seu cinturão protetor para explicar anomalias e refutações de suas hipóteses, ele passa a receber os ataques diretamente. Isto expõe sua heurística negativa a teste, podendo resultar em sua substituição por um programa concorrente. De acordo com Popper (1975), o projeto de pesquisa refutado empi-

\footnotetext{
${ }^{9}$ Interessante notar que Thaler usa o termo "anomalia”, o mesmo usado por Kuhn em sua teoria de revoluções científicas. Resta saber, porém, se vai haver massa crítica de persuasão na comunidade científica da área de economia sobre sua importância. Uma busca no banco de textos Econpapers em janeiro de 2009 gerou 1189 hits com a palavra "anomalies" no título ou no resumo e 523 hits com "prospect theory". Como simples referências, fizemos a pesquisa com o termo "transaction cost economics" (1443 hits) e com o termo "bounded rationality" (926 hits).

${ }^{10}$ Além dos trabalhos já citados na introdução, ver também Lewin (1996), Binmore (1999) e Davis (2008b). A visão interna à economia experimental pode ser encontrada em, e.g., Camerer et al. (2003) e Carbone \& Starmer (2007). Para visões internas à teoria da perspectiva em particular, ver Kahneman e Tversky (2000) e Thaler (2000).
} 
ricamente será substituído se houver uma alternativa metodologicamente viável e que também possa ser falseável.

Kuhn $(1962,1979)$ ressaltaria que a refutação empírica apenas não determinaria uma revolução científica e a troca de paradigmas. Seria necessária também a existência de um sentimento de "crise" entre os cientistas, ou de reconhecimento disseminado e persistente de alguma "anomalia" (Kuhn, 1977, p. 203). No que se refere ao caso aqui discutido, é difícil dizer se há uma revolução em andamento. Algumas evidências de mudança são detectáveis, como apresentadas anteriormente, mas seu alcance e sua capacidade de fixação e disseminação ainda são difíceis de dimensionar. Talvez só a distância histórica nos permita montar um argumento mais plausível a este respeito, como o fez Kuhn em seus mais fundamentados exemplos de revoluções científica. ${ }^{11}$ Por isso nos detemos a situar este estudo num momento de possível transição, mas que não deixa claro se para um novo paradigma dominante ou para um ambiente mais plural de programas de pesquisa divergentes (Davis, 2006).

Assim, discutiremos a seguir em que pontos a teoria da perspectiva se mostra alternativa aos modelos racionais. Para tanto vamos delinear as teorias racionais de tomada de decisão sob risco e a teoria da perspectiva em acordo com o referencial lakatoseano de programas de pesquisa com vistas a compará-las.

\section{Teorias racionais de decisão sob risco}

Entre as teorias racionais de decisão sob risco, podemos situar a Teoria dos Jogos de Von Neumann e Morgenstern, a Teoria de Alocação de Portfólio de Markowitz, e o CAPM (Capital Asset Pricing Model) de Sharpe. Embora haja diferenças em seus modelos e aplicações, seus pressupostos principais são idênticos: a ideia de que os agentes podem formular uma distribuição de probabilidades dos eventos e conhecer seus respectivos pay-offs, e maximizam o saldo de suas escolhas em consonância com a teoria da utilidade esperada.

A teoria da utilidade se sustenta em quatro suposições básicas: cancelamento, transitividade, dominância e invariância. De acordo com o princípio da dominância, se determinada opção é melhor que outra em um dos possíveis resultados, e pelo menos igual em todas as outras possibilidades, então a opção dominante deve ser escolhida. A condição de invariância é respeitada quando a preferência entre uma escolha ou outra é independente da maneira como elas são apresentadas. $\mathrm{O}$ cancelamento é a propriedade de eliminar do processo decisório outros eventos que gerem os mesmos resultados, independente da escolha realizada. A transitividade possibilita representar a preferência por diferentes opções com uma escala ordinal, uma escala que será respeitada independente das opções comparadas: se A é preferível a B e B é preferível a $C, C$ não pode ser preferível a $A$. Essa suposição é

\footnotetext{
${ }^{11}$ Como ressalta Muramatsu (2009), muitos economistas tentam seguir uma estratégia de mudanças graduais. Há, assim, um esforço em acomodar novas ideias às bases teóricas que dominam e com as quais se sentem seguros, e isto tem acontecido com os economistas que se utilizam de teorias das decisões e se deparam com as novidades propostas pela economia experimental.
} 
respeitada quando é possível determinar um valor para cada opção de escolha e que esse valor não dependa das outras (Kahneman \& Tversky, 1986).

De acordo com Kahneman e Tversky (1986), os pressupostos de cancelamento e transitividade foram relaxados no desenvolvimento de modelos racionais de escolha sob risco, com a alteração da função de utilidade que passou a ser não linear e bivariável. Por isso nós não as consideramos aqui parte do núcleo duro das teorias racionais de escolha.

O mesmo não acontece com os pressupostos de dominância e invariância, intimamente ligados à ideia de racionalidade substantiva. A invariância em especial é tacitamente assumida na caracterização de opções em vez de ser uma hipótese testável (Kahneman \& Tversky, 1986). Muitos dos modelos racionais descrevem as opções de escolha como variáveis aleatórias, e representações alternativas das mesmas não são tratadas como tendo influência no processo decisório.

De acordo com Kahneman e Tversky (1979), para que a teoria da utilidade esperada possa ser aplicada a escolhas, três princípios precisam ser adicionados: expectativa, integração com ativos e aversão ao risco. Pelo princípio da expectativa a utilidade total de qualquer prospecto (um conjunto de possíveis resultados e suas chances de ocorrerem a partir de uma escolha) é igual à soma das utilidades esperadas dos seus possíveis resultados. Pelo princípio da integração com ativos, um certo prospecto só é aceitável se a utilidade de integrá-lo aos ativos do agente superarem a utilidade destes ativos, i.e., a função utilidade trata de estados finais de riqueza e não de ganhos e perdas. Pelo princípio da aversão ao risco, o agente deve preferir um prospecto com um resultado certo " $\mathrm{x}$ " a qualquer prospecto de risco cujo valor esperado é o mesmo " $\mathrm{x}$ ".

Deriva-se assim que, de acordo com os autores, o núcleo duro das teorias modernas de decisão sob risco conta com os seguintes pressupostos:

i) uma etapa no processo decisório: não há separação (e portanto qualquer atrito) entre absorção de informações, processamento das mesmas, confecção das distribuições de probabilidade dos eventos e elaboração dos respectivos pay-offs ligados ao espectro de ações disponíveis. $\mathrm{O}$ processo gera por princípio um pay-off ótimo a se escolher;

ii) vigoram os axiomas de dominância e invariância;

iii) valem os princípios da expectativa, da integração com ativos, e da aversão ao risco.

Em complemento, fazem parte de seu cinturão protetor as ideias derivadas desses princípios e que são expostas aos testes empíricos, que são:

i) a lineariedade da ponderação das utilidades de cada opção de escolha por sua probabilidade - base para o cancelamento e para a transitividade (Kahneman \& Tversky, 1979; Camerer, 1998);

ii) a avaliação de resultados como estados finais de riqueza; e

iii) da aversão ao risco, a concavidade da curva de utilidade (medida em estados finais de riqueza).

Essa identificação é uma base comum aos modelos racionais de tomada de decisão de cunho normativo. Espera-se que as críticas à utilização destes modelos como descritivos e a apresentação de inconsistências com a realidade através de experimentos levem seus praticantes a ocasionalmente relaxar alguma das hipóte- 
ses de seu programa de pesquisa. Isto, porém, costuma se dar dentro do cinturão de proteção, como detectaram Kahneman e Tversky para o caso do cancelamento e transitividade, e não dos pressupostos do núcleo duro - a não ser, claro, que se queira questionar o programa.

\section{Teoria da perspectiva}

Segundo Kahneman e Tversky (1986), a teoria da perspectiva é consistente com a ideia de racionalidade limitada proposta e disseminada por Herbert Simon (1957). A racionalidade limitada e seus fundamentos de ordem psicológica do processo decisório são parte importante da refutação da racionalidade substantiva. Todo experimento da teoria da perspectiva assume, ab initio, que há limites de racionalidade. Não fosse assim, os experimentos não fariam sentido - os resultados poderiam ser deduzidos aprioristicamento pelo formulador do experimento. Assim, alguns princípios marcantes da noção de racionalidade limitada fazem parte de seu núcleo rígido, em contraste com as bases da noção de racionalidade substantiva.

Fazem parte da heurística negativa da teoria da perspectiva, por exemplo, o princípio da ponderação não-linear de eventos possíveis através de pesos de decisão e a divisão do processo decisório em duas fases.

Os pesos de decisão são uma função da chance de certo resultado acontecer, mas não são uma probabilidade em si nem são lineares, contrariando o princípio da expectativa e suas implicações. As pessoas os utilizam para ponderar os possíveis resultados ao invés de utilizar a probabilidade pura, como nos modelos racionais. Tais pesos estão sujeitos à influência de efeitos psicológicos diversos mas sistemáticos, e são passíveis de detecção nos experimentos na forma de vieses e heurísticas (Kahneman \& Tversky, 1986; Camerer \& To, 1999).

A estrutura cognitiva dos agentes faz com que o processo decisório seja fragmentado. Na teoria da perspectiva isso é caracterizado por uma divisão em duas fases: uma de enquadramento ou montagem (framing) e uma subsequente de avaliação de resultados. Na primeira fase os agentes visualizam o problema, analisando as possíveis escolhas, contingências e resultados. Nesta fase eles eliminam componentes comuns às diferentes opções e também opções que pareçam dominadas por outras, montando um "prospecto". O prospecto em si é, portanto, resultado tanto da maneira como o problema é apresentado ao agente, quanto de seus hábitos e modelos mentais, normas e expectativas. $\mathrm{Na}$ segunda fase, as opções do prospecto são avaliadas e o indivíduo escolhe a que lhe apresentar maior valor (Kahneman \& Tversky, 1986).

Por fim, a teoria da perspectiva assume que os indivíduos não determinam o valor de uma escolha de acordo com os possíveis estados finais de riqueza, mas sim em comparação a um ponto de referência. Ou seja, as escolhas são analisadas de acordo com possíveis ganhos ou perdas relativos a tal baliza. A teoria também assume que o valor negativo atribuído a perdas é maior que o valor positivo atribuído a ganhos de mesma magnitude, o que denominaram de aversão à perda loss aversion (Kahneman et al. 1991). Assim, a função de utilidade tem a forma de um "S", côncava para ganhos e convexa para perdas. 
No cinturão de proteção da teoria da perspectiva estão seus elementos mais expostos a testes. Assim, das ideias de ponderação não-linear dos pesos de decisão e das diferenças causadas pelo enquadramento, identificamos os resultados possivelmente refutáveis do efeito certeza e do efeito pseudocerteza, que demonstram que resultados certos são sobrevalorizados. Derivam das mesmas ideias as falhas de invariância, quando apresentações diferentes de um mesmo problema resultam em preferências diferentes, o que em algumas ocasiões também gera falhas de dominância (Kahneman \& Tversky, 1986). Das ideias de que os agentes avaliam resultados em termos de ganhos e perdas, e de que são avessos à perda, têm-se os efeitos de dotação e pró-situação - o indivíduo dá mais valor a bens que já possui do que aos que não possui, e de forma mais ampla a situações que já estão postas do que a situações diferentes (Kahneman et al. 1991).

\section{Um prospecto comparativo lakatoseano}

A Figura 2 ilustra a comparação entre as teorias racionais de decisão sob risco e a teoria da perspectiva nos termos lakatoseanos a que recorremos. (Ver esquema abaixo).

Figura 2: Esquema comparativo
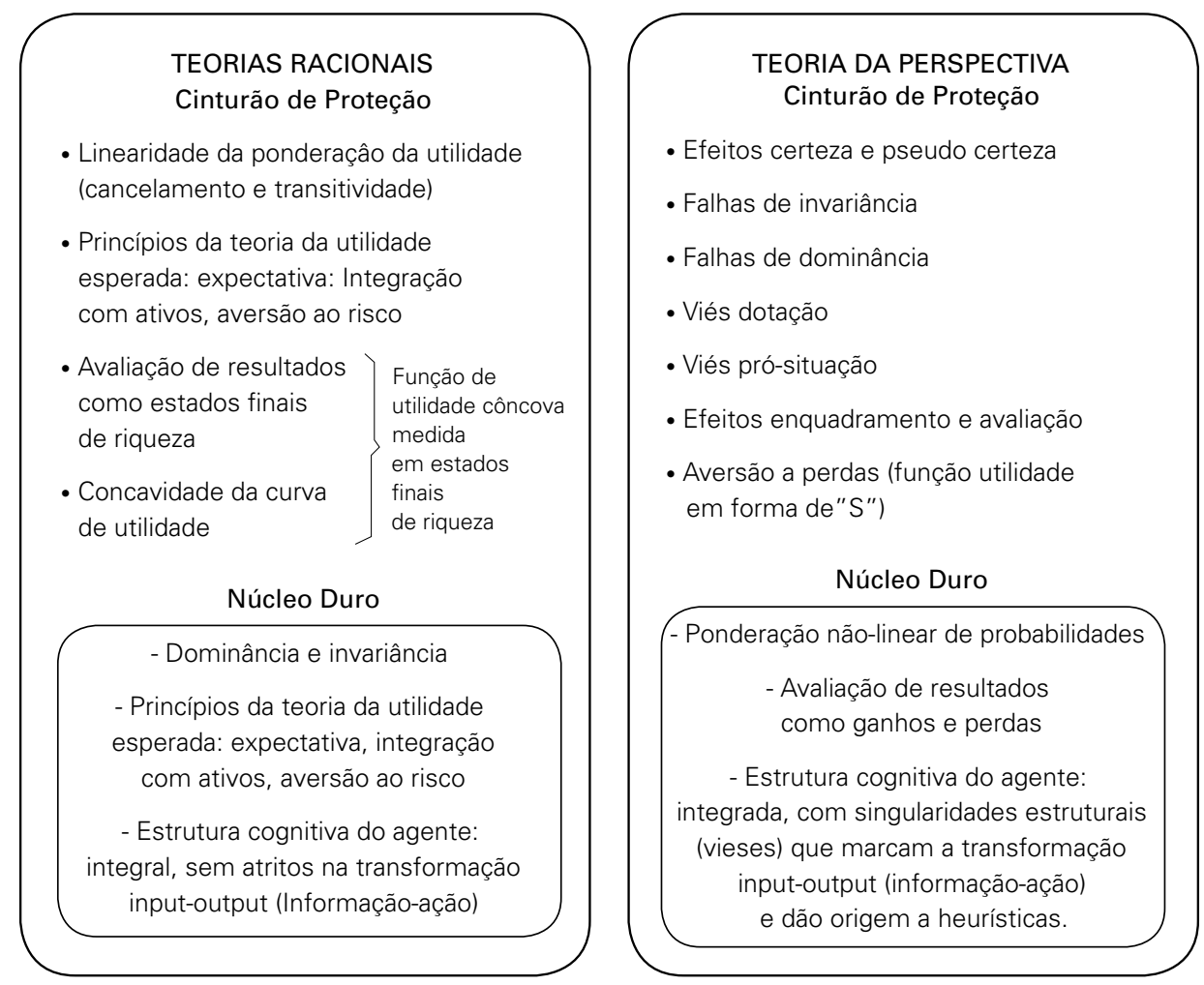

Fonte: Elaboração própria 
Se os argumentos apresentados são razoáveis, pode-se ver mais facilmente na Figura 2 que a teoria da perspectiva contraria o poder descritivo das teorias normativas e confronta não apenas sua heurística positiva mas, mais importante, o seu núcleo duro. Um ponto a se observar é que as contrapartidas das ideias do cinturão de proteção das teorias racionais de decisão são parte do núcleo duro da teoria da perspectiva. Isso se deve a diferenças estruturais de concepção destas teorias. Como as teorias racionais surgem na forma de explicações lógicas sobre como um agente idealizado toma decisões dentro dos modelos (ou seja, teorias normativas), seu núcleo duro é basicamente composto de pressupostos que sustentam a noção de racionalidade substantiva. A teoria da perspectiva surge a partir da crítica à falta de conexão com a realidade de tais pressupostos e da observação experimental. Assim, seu núcleo duro tem regularidades empíricas (experimentais) que dão suporte à noção de racionalidade limitada. Ele parte de um conjunto de regularidades manifestas das quais são deduzidas certas estruturas cognitivas da mente humana. Por conseguinte, seu cinturão de proteção contém basicamente "efeitos", fenômenos e padrões observados em testes mais específicos, e explicados pelas estruturas cognitivas definidas na heurística negativa.

A comparação reflete as diferenças entre um sistema axiomático puro, inspirado na física clássica (Mirowski, 1989), e outro indutivo, inspirado nas práticas da psicologia. As teorias racionais de tomada de decisão criam modelos de tomada de decisão normativos, ideais, baseados em princípios lógicos, enquanto a teoria da perspectiva observa desvios sistemáticos daqueles e os generaliza no núcleo duro. É a partir deles que se criam modelos mentais que sejam capazes de identificar efeitos mais específicos (os desvios ou anomalias) e descrevê-los logicamente. Mas como tais diferenças podem indicar mudanças nas preferências profissionais de economistas acadêmicos envolvidos com o estudo do processo decisório individual?

\section{TEORIA DA PERSPECTIVA E AS PERSPECTIVAS DA CIÊNCIA ECONÔMICA}

O mainstream neoclássico se mostrou resistente à crítica interna que, de longa data, se dirigia tanto à rigidez e unicidade do método hipotético-dedutivo quanto à natureza axiomática e universal de seus fundamentos. Mas são fontes externas à sua bacia que vêm mudando seu curso. Experimentos, comuns à psicologia, tanto quanto simulações e métodos não lineares, mais comuns à biologia e à computação, têm tido mais espaço no trabalho acadêmico dos economistas. A teoria da perspectiva em particular oferece espaço para estudos de situações que são dependentes de contexto e que, portanto, não precisam se basear em premissas axiomáticas universais (Hodgson, 2007a,b; Pressman, 2006). Além disso, ela permite (não sem controvérsias) através do método experimental instrumentalizar hipóteses e testálas - algo tido por impossível e desejável por autoridades da área como Milton Friedman (1953) e Paul Samuelson (Samuelson \& Nordhaus, 1985).

Este é um campo de interesse de correntes do pensamento à margem do mains- 
tream neoclássico. Davis (2006, 2008a), Hodgson (2007) e Pressman (206), por exemplo, sugerem as teorias evolucionária e institucional como campos conexos a tais desenvolvimentos. Isso lhes permite imaginar um futuro possível em que o mainstream será mais plural em vez de contar com uma abordagem dominante.

Economistas são famosos por suas previsões frustradas, mas a construção de cenários é sua sina. Se o cenário de maior pluralismo é razoável, então vale discutir o que a teoria da perspectiva pode oferecer.

Uma questão relevante é a de sua ascendência à teoria econômica. Se a teoria da decisão racional serviu de base a uma teoria econômica neoclássica com derivações importantes na micro e na macroeconomia, como pode a teoria da perspectiva se engajar na produção do pensamento econômico ${ }^{12}$ Como Matthew Rabin (2008) identifica, a teoria da perspectiva se insere num programa de pesquisa de heurísticas e vieses de julgamento. Há ali, acima de tudo, um imperativo metodológico: a busca, via indução, de padrões de comportamento. A teoria da perspectiva identifica alguns e incentiva a busca por outros. $\mathrm{O}$ método axiomático da teoria neoclássica parece mais facilmente permissivo a uma sistematização teórica mais homogênea e de aplicação menos custosa. Isto facilita o trabalho do arms-chair economist fazendo "blackboard economics" (Coase, 1994) e lidando com dados secundários. Não é o que se vê acontecer com a teoria da perspectiva. Em lugar de tomar como dado o comportamento universal do indivíduo, o economista tem que trabalhar antes na identificação de heurísticas e vieses de comportamento. Com isso ele precisará lidar com pelo menos duas novidades. Uma delas é uma nova orientação metodológica para lidar com a indução experimental. A outra é a necessidade de recursos para a realização de, e.g., experimentos, surveys e surveys hipotéticos. ${ }^{13}$

Uma diferença interessante vale ser notada. Com a teoria neoclássica, todas as decisões dos indivíduos são passíveis de racionalização ex post como conduta compatível com a teoria. Com a teoria da perspectiva há espaço para a incerteza. Se por exemplo o pesquisador adota a priori a hipótese de algum viés (e.g., dotação ou pró-situação) e isto passa a ser de conhecimento dos agentes estudados, podem eles agir de modo diferente na tentativa de "neutralizar" o viés? Ou seja, não parece viável que a teoria da perspectiva entre no pensamento econômico na forma de uma microeconomia universal cujos princípios se apliquem uniformemente aos consumidores, aos investidores ou às firmas. Parece mais razoável que isto se dê de forma mais fragmentada a partir de experiências empíricas localizadas que sirvam de referência para emulações em outros contextos.

Como sugere Davis (2008a), a teoria da perspectiva oferece uma forma "sin-

\footnotetext{
${ }^{12}$ Agradecemos a um parecerista por ter levantado este ponto.

${ }^{13}$ Vale notar aqui a virtual nulidade da pesquisa econômica brasileira no campo. Talvez a predominância de interesse na macroeconomia faça mais rarefeita a preferência dos economistas brasileiros pela experimentação microeconômica possibilitada pela teoria da perspectiva, bem como pela possibilidade de explorar peculiaridades da cultura e do contexto brasileiros no que tange às decisões econômicas.
} 
crônica" (e não "diacrônica") de explicação. Seu fenômeno de estudo tem uma extensão temporal limitada na qual dificilmente se dá atenção a processos de mudança e formação de preferências em si. Um possível desdobramento disto é que, a partir de estudos de decisões influenciáveis por hábitos variados, estudos comparativos possam ser feitos - e.g., entre hábitos de diferentes profissões, gerações, nacionalidades ou gêneros (Carpenter et al., 2005). Isto permitiria explorar efeitos de enquadramento social das decisões dos indivíduos (Klaes, 2008). Pode-se ainda explorar mudanças no tempo a partir de experimentos repetidos. ${ }^{14}$

A princípio, tais desdobramentos fazem emergir a possibilidade de se encontrar regularidades peculiares, institucional e historicamente dependentes. Isto colocaria em xeque o próprio o núcleo rígido da teoria da perspectiva. Talvez, neste caso, se possa ter um núcleo soft que comporte hipóteses duais (e.g., avaliação de resultados em termos de ganhos e perdas ou em termos de status posicional), ou um núcleo duro com imperativos meta-teórico (e.g., a experimentação que satisfaça tal conjunto de condições identifica o tipo de avaliação de resultados que caracteriza os indivíduos de um estudo).

\section{CONSIDERAÇÕES FINAIS}

A pesquisa moderna sobre o processo decisório em grande parte tenta detectar, contestar e superar as deficiências das teorias racionais, tal como exemplificam o paradoxo de Allais e seus desdobramentos no campo da economia. A teoria da perspectiva dos psicólogos Daniel Kahneman e Amos Tversky se mostra um desenvolvimento importante nesta área. Ela vem conseguindo espaço na prática dos economistas ao lidar com diversos comportamentos não explicados pelas teorias racionais. Kahneman e Tversky, em especial, se voltaram a desenvolver uma teoria descritiva do processo decisório com base em experimentos, o que destoa das teorias tradicionais da escolha - normativas e axiomáticas.

Ao enquadrarmos os modelos racionais de escolha e a teoria da perspectiva num esquema lakatoseano, a comparação entre suas estruturas metodológicas evidencia o teor de suas diferenças. As teorias racionais têm o que mais se aproxima de estruturas cognitivas e bases para a tomada de decisão como heurística positiva, deixando princípios axiomáticos apriorísticos para fundamentá-las. Já a teoria da perspectiva tem estruturas cognitivas e bases para a tomada de decisão em sua heurística negativa, apreendidas da observação do comportamento individual em experimentos. Sua heurística positiva é constituída basicamente de efeitos e padrões relacionados a situações experimentais mais específicas. De tal modo, a teoria da perspectiva tem conseguido criar um corpo teórico descritivo do processo de escolha de maneira diferente das teorias já postas sobre o assunto.

\footnotetext{
${ }^{14}$ Modelos econométricos de painel não balanceado, por exemplo, podem usar experimentos repetidos ao longo do tempo ou em contextos diferentes (cross-section).
} 
Várias possibilidades de pesquisa foram abertas pela teoria da perspectiva, tanto teóricas quanto empíricas. Pelo lado teórico, discute-se, por exemplo, a validade e a aplicabilidade dos resultados experimentais. Deste tema derivam discussões sobre a condução de experimentos, e.g., maneiras de obter maior controle em laboratórios e melhor determinação de relações causais. Discute-se também a natureza de cada efeito ou viés, o que pode dar espaço para uma visão mais "diacrônica” e também social dos fenômenos estudados. Isto também tem o potencial de aproximar a teoria da pespectiva de outras abordagens mais institucionais e evolucionárias.

Pelo lado empírico, a identificação de "anomalias" ou de padrões peculiares de comportamento em estudos de caso tem se espalhado. Experimentos comparativos de processos decisórios com vistas a identificar influências culturais e institucionais tanto sincrônicas quanto diacrônicas começam a surgir. Isto pode ser de interesse direto a microeconomistas brasileiros - uma vereda teórica que dê mais liberdade ao estudo de peculiaridades diversas do comportamento dos brasileiros.

Por fim, é ainda difícil vislumbrar o que será o mainstream econômico no futuro próximo. As novas gerações de economistas encontram certamente um rol diferente de oportunidades de pesquisa no que se costuma delimitar como microeconomia. O papel da teoria da perspectiva na mudança de preferências teóricas dos economistas dependerá em parte de como a atual geração de acadêmicos expuser suas conquistas e potencialidades, e de como os mais novos as vão avaliar — uma questão de enquadramento e avaliação.

\section{REFERÊNCIAS BIBLIOGRÁFICAS}

BIANCHI, A. M. (2001). "Economistas de avental branco: uma defesa do método experimental na economia." Revista de Economia Contemporânea 5(2):129-54.

BINMORE, K. (1999). “Why experiment in economics?” Economic Journal 109: F16-24.

BOLAND, L. (1981). "On the futility of criticizing the neoclassical maximization hypothesis." American Economic Review 71:1031-6.

CAMERER, C. (1998). "Bounded rationality in individual decision making." Experimental Economics $1(2): 163-83$.

CAMERER, C. \& HO, T. (1999). "Experience-weighted attraction learning in normal form games." Econometrica 67(4):827-74.

CARBONE, E. \& STARMER, C. (eds) (2007). New developments in experimental economics. Farmington Hills: Edward Elgar, 2 volumes.

CARPENTER, J. \& BURKS, S \& VERHOOGEN, E. (2005). “Comparing students to workers: the effects of social framing on behavior in distribution games.” In CARPENTER, J. \& LIST, J. \& HARRISON, G. (eds). Field experiments in economics. Greenwich: JAI Press, pp. 261-90.

COASE, R. (1994). Essays on Economics and economists. Chicago: University of Chicago Press.

COLANDER, D. (2000). "The death of neoclassical economics." Journal of the History of Economic Thought 22(2):127-43.

COLANDER, D. \& HOLT, R. \& ROSSER JR, B. (2004). "The changing face of mainstream economics.” Review of Political Economy 16(4):485-99.

DAVIS, J. B. (2006). "The turn in economics: neoclassical dominance to mainstream pluralism?” Journal of Institutional Economics 2:1-20. 
DAVIS, J. B. (2008a). “The turn in recent Economics and return of orthodoxy.” Cambridge Journal of Economics 32(3):349-66.

DAVIS, J. B. (2008b). "Psychology's recent challenge to economics: Rationality and the individual." Texto apresentado na History of Economics Society 2008 Annual Conference, Toronto.

DEQUECH, D. (2007). "Neoclassical, mainstream, heterodox, and orthodox economics." Anais do XXXV Encontro Nacional de Economia, Recife.

FRIEDMAN, D. (1998). "Evolutionary economics goes mainstream: a review of the theory of learning in games." Journal of Evolutionary Economics 8(4):423-32.

FRIEDMAN, M. (1953). Essays in positive economics. Chicago: University of Chicago Press.

GLIMCHER, P. (2002). "Decisions, decisions, decisions: choosing a biological theory of choice." Neuron 36:323-32.

GUALA, F. (2002). "On the scope of experiments in economics: comments on Siakantaris." Cambridge Journal of Economics 26(2):261-7.

HANDS, D. W. (2007). "Economics, psychology, and the history of consumer choice theory." Manuscrito. Disponível em: <http://ssm.com/abstract=988125> Acesso em: 28 de agosto de 2007.

HODGSON, G. (2007a). "Evolutionary and institutional economics as the new mainstream?" Evolutionary and Institutional Economics Review 4(1):7-25.

HODGSON, G. (2007b). “The revival of Veblenian Institutional Economics.” Journal of Economic Issues 41(2):325-40.

HUTCHISON, T. W. (1938). The significance and basic postulates of economic theory. Londres: Macmillan.

KAGEL, J. \& ROTH, A. (eds) (1995). The handbook of experimental economics. Princeton: Princeton University Press.

KAHNEMAN, D. \& KNETSCH, J. \& THALER, R. (1990). "Experimental tests of the endowment effect and the Coase theorem." Journal of Political Economy 98:1325-48.

KAHNEMAN, D. \& KNETSCH, J. \& THALER, R. (1991). "Anomalies: the endowment effect, loss aversion, and status quo bias." Journal of Economic Perspectives 5(1):193-206.

KAHNEMAN, D. \& TVERSKY, A. (1979). "Prospect theory: an analysis of decision under risk." Econometrica 47(2):263-91.

KAHNEMAN, D. \& TVERSKY, A. (1986). "Rational choice and the framing of decisions." The Journal of Business 59(4):251-78.

KAHNEMAN, D. \& TVERSKY, A. (2000). Choices, values, and frames. Nova Iorque: Cambridge University Press.

KLAES, M. (2008). "Rationality and its bounds: re-framing social framing." In Galavotti, M. \& Suppes, P. \& Scazzieri, R. (eds). Reasoning, rationality and probability. Chicago: Chicago University Press, pp. 211-32.

KUHN, T. S. (1962). The structure of scientific revolutions. Chicago: University of Chicago Press.

KUHN, T. S. (1977). The essential tension. Chicago: University of Chicago Press.

KUHN, T. S. (1979). "Lógica da descoberta ou psicologia da pesquisa?” In LAKATOS, I. \& MUSGRAVE, A. (orgs). A crítica e o desenvolvimento do conhecimento. São Paulo: Cultrix, pp. 5-32.

LAKATOS, I. (1979). "O Falseamento e a metodologia dos programas de pesquisa científica." In LAKATOS, I. \& MUSGRAVE, A. (orgs). A crítica e o desenvolvimento do conhecimento. São Paulo: Cultrix, pp. 109-243.

LEWIN, S. B. (1996). "Economics and Psychology: lessons for our own day from the early Twentieth Century." Journal of Economic Literature 34:1293-1323.

MONGIN, P. (1988). "Expected utility theory." In DAVIS, J. \& HANDS, D. W. \& MÄKI, U. (eds). Handbook of economic methodology. Londres: Edward Elgar, pp. 342-50.

MURAMATSU, R. (2009). "The death and resurrection of 'economics with psychology': remarks from a methodological standpoint." Revista de Economia Politica 29(1).

PLOTT, C. (1991). “Will economics become an experimental Science?” Southern Economic Journal 57(4):901-19. 
POPPER, K. (1975). A lógica da investigação científica. Coleção Os Pensadores. São Paulo: Abril Cultural.

PRESSMAN, S. (2006). "Kahneman, Tversky, and institutional economics” Journal of Economic Issues 40(2):501-6.

RABIN, M. (2008). “"Kahneman, Daniel (born 1934).” In DURLAUF, S. \& BLUME, L. (eds). The New Palgrave Dictionary of Economics Online. Second Edition. Palgrave Macmillan. Disponível em http://www.dictionaryofeconomics.com/article?id=pde 2008_K000065. Acesso em: 20 de dezembro de 2008.

RSAS - THE ROYAL SWEDISH ACADEMY OF SCIENCES (2002). "Advanced Information on the Prize in Economic Sciences - Foundations of Behavioral and Experimental Economics: Daniel Kahneman and Vernon Smith.” Disponível em: <http:// nobelprize.org/nobel_prizes/economics/ laureates/2002/ecoadv02.pdf>. Acesso em: 05 de setembro de 2007.

SAMUELSON, P. \& NORDHAUS, W. (1985). Economics. 12 ${ }^{\mathrm{a}}$ ed. Nova Iorque: McGraw-Hill.

SENT, E.-M. (2004). "Behavioral economics: how Psychology made its (limited) way back into Economics." History of Political Economy 36:735-60.

SIAKANTARIS, N. (2000). "Experimental economics under the microscope." Cambridge Journal of Economics 24(3):267-81.

SIMON, H. A. (1957). Models of man. New York: John Wiley.

SMITH, V. (1962). “An experimental study of competitive market behavior.” Journal of Political Economy 70:111-37.

SMITH, V. L. (1982). "Microeconomic systems as an experimental science.” American Economic Review 72(5):923-55.

SMITH, V. L. (1990). "Experimental economics.” Aldershot: Edward Elgar.

SMITH, V. L. (2002). "Method in experiment: rhetoric and reality.” Experimental Economics 5(2):91110.

THALER, R. (2000). "From homo economicus to homo sapiens.” Journal of Economic Perspectives $14(1): 133-41$.

THALER, R. \& TVERSKY, A. \& KAHNEMAN, D. \& SCHWARTZ, A. (1997). "The effect of myopia and loss aversion on risk taking: an experimental test." Quarterly Journal of Economics 112:647-61.

TVERSKY, A. \& KAHNEMAN, D. (1974). "Judgment under uncertainty: heuristics and biases." Science 185(4157):1124-31.

TVERSKY, A. \& KAHNEMAN, D. (1992). “Advances in Prospect Theory: cumulative representation of uncertainty." Journal of Risk and Uncertainty 5(4):297-323.

VEBLEN, T. (1898). "Why is economics not an evolutionary science?" The Quarterly Journal of Economics 12(4):373-97.

VILLEVAL, M. C. (2007). "Experimental economics: contributions, recent developments, and new challenges.” Working Paper 07-06: GATE-Groupe d'Analyse et de Théorie Économique.. 